\title{
Asymptotic behavior of solutions to a class of coupled semilinear parabolic systems with gradient terms
}

\author{
Yang $\mathrm{Na}^{\mathrm{a}}$, Yuanyuan $\mathrm{Nie}^{\mathrm{a}}, \mathrm{Xu} \mathrm{Zhou}^{\mathrm{b}, *}$ \\ ${ }^{a}$ School of Mathematics, Jilin University, Changchun 130012, P. R. China. \\ ${ }^{b}$ College of Computer Science and Technology, Jilin University, Changchun 130012, P. R. China.
}

\begin{abstract}
This paper concerns the asymptotic behavior of solutions to the Cauchy problem of a class of coupled semilinear parabolic systems with gradient terms. Using the energy comparison method and comparison principle, the blow-up theorem of Fujita type is established and the critical Fujita curve is formulated by spacial dimension, the behavior of the coefficient of the gradient term at infinity. (C)2017 All rights reserved.
\end{abstract}

Keywords: Asymptotic behavior, critical Fujita curve, gradient term. 2010 MSC: 35B33, 35K20, 35K58.

\section{Introduction}

We investigate the asymptotic behavior of solutions to the Cauchy problem of coupled semilinear parabolic systems of the form

$$
\begin{array}{ll}
\frac{\partial u}{\partial t}=\Delta u+b(|x|) x \cdot \nabla u+v^{p}, & x \in \mathbb{R}^{n}, t>0, \\
\frac{\partial v}{\partial t}=\Delta v+b(|x|) x \cdot \nabla v+u^{q}, & x \in \mathbb{R}^{n}, t>0, \\
u(x, 0)=u_{0}(x), \quad v(x, 0)=v_{0}(x), & x \in \mathbb{R}^{n},
\end{array}
$$

where $p, q>1,0 \leqslant b \in C^{1}([0,+\infty))$ satisfies

$$
\lim _{s \rightarrow+\infty} s^{2} b(s)=k, \quad(0 \leqslant k \leqslant+\infty),
$$

and $0 \leqslant u_{0}, v_{0} \in \mathrm{L}_{\text {loc }}^{1}\left(\mathbb{R}^{\mathfrak{n}}\right) \cap \mathrm{L}^{\infty}\left(\mathbb{R}^{\mathrm{n}}\right)$ are nontrivial.

It is well-known that the Cauchy problem of the semilinear equation

$$
\frac{\partial u}{\partial t}=\Delta u+u^{p}, \quad x \in \mathbb{R}^{n}, t>0,
$$

was investigated by Fujita in [3], where it was proved that the problem does not have any nontrivial global nonnegative solution if $1<p<p_{c}=1+2 / n$, whereas there exist both nontrivial global (with small initial

\footnotetext{
*Corresponding author

Email address: zhouxu0001@163.com (Xu Zhou)
} 
data) and non-global nonnegative (with large initial data) solutions if $p>p_{c}$. Furthermore, for the critical case $p=p_{c}$, it was shown in $[6,7,19]$ that the problem possesses no nontrivial global nonnegative solution. Later, there have been a number of extensions of Fujita's results in several directions, including similar results for numerous of nonlinear parabolic equations and systems (see, e.g., the survey papers $[1,2,4,5,8-12,14-18,20-23]$ and the references therein).

Among those, there are a few studies on equations and systems with gradient terms. It is Meier [10] who first studied the critical Fujita exponent for the Cauchy problem

$$
\frac{\partial u}{\partial t}=\Delta u+\vec{b}(x) \cdot \nabla u+u^{p}, \quad x \in \mathbb{R}^{n}, t>0,
$$

with $\vec{b} \in L^{\infty}\left(\mathbb{R}^{n} ; \mathbb{R}^{n}\right)$. It was shown that

$$
p_{c}=1+\frac{1}{\gamma^{*}}
$$

where $\gamma^{*}$ is the maximal decay rate for solutions to

$$
\frac{\partial w}{\partial t}=\Delta w+\vec{b}(x) \cdot \nabla w, \quad x \in \mathbb{R}^{n}, t>0
$$

If $\vec{b}$ is constant, it is clear that $\gamma^{*}=n / 2$ and $p_{c}=1+2 / n$. However, for nonconstant $\vec{b} \in L^{\infty}\left(\mathbb{R}^{n} ; \mathbb{R}^{n}\right), \gamma^{*}$ and $p_{c}$ are unknown generally. It is noted that [21] studied the case that

$$
\vec{b}(s)=\frac{\kappa}{|s|^{2}}, \quad s \in \mathbb{R}^{n}, \quad(-\infty<\kappa<+\infty) .
$$

Since such a function is singular at 0 when $k \neq 0$, the authors considered the following Neumann exterior problem

$$
\begin{array}{ll}
\frac{\partial u}{\partial t}=\Delta u+\frac{\kappa}{|x|^{2}} x \cdot \nabla u+u^{p}, & x \in \mathbb{R}^{n} \backslash B_{1}, t>0, \\
\frac{\partial u}{\partial v}(x, t)=0, & x \in \partial B_{1}, t>0, \\
u(x, 0)=u_{0}(x), & x \in \mathbb{R}^{n} \backslash \bar{B}_{1},
\end{array}
$$

and showed that its critical Fujita exponent can be formulated as

$$
p_{c}= \begin{cases}1, & \kappa=+\infty, \\ 1+2 /(n+\kappa), & -n<\kappa<+\infty, \\ +\infty, & -\infty \leqslant \kappa \leqslant-n,\end{cases}
$$

where $B_{1}$ is the unit ball in $\mathbb{R}^{n}$ and $v$ is the unit inner normal vector to $\partial B_{1}$. Moreover, $[18,21]$ also investigated the Neumann exterior problems for the Newtonian and non-Newtonian filtration equations with similar gradient terms. [23] showed that the critical Fujita exponent to the Cauchy problem (1.5) with $\vec{b}=b$ is still (1.6), where b satisfies (1.4). Furthermore, Suzuki [14] considered the Newtonian filtration equation with similar gradient term and got the critical Fujita exponent for some special cases.

In 1991, Escobedo and Herrero [2] investigated the coupled semilinear parabolic system (1.1), (1.2), (1.3) in the special case $b \equiv 0$, and formulated the critical Fujita curve as

$$
(p q)_{c}=1+\frac{2}{n} \max \{p+1, q+1\} .
$$

The authors in [4] considered the Neumann exterior problem of coupled nonlinear diffusion systems

$$
\frac{\partial u}{\partial t}=\Delta u+\frac{k}{|x|^{2}} x \cdot \nabla u+|x|^{\lambda} v^{p}, \quad x \in \mathbb{R}^{n} \backslash B_{1}, t>0,
$$




$$
\frac{\partial v}{\partial t}=\Delta v+\frac{\kappa}{|x|^{2}} x \cdot \nabla v+|x|^{\lambda} u^{q}, \quad x \in \mathbb{R}^{n} \backslash B_{1}, t>0,
$$

where $\kappa \in \mathbb{R}, \lambda \geqslant 0$, and proved its critical Fujita curve

$$
(p q)_{c}= \begin{cases}1+\frac{2+\lambda}{n+k} \max \{p+1, q+1\}, & \kappa>-n, \\ +\infty, & \kappa \leqslant-n .\end{cases}
$$

In the present paper, we prove that the critical Fujita curve to the problem can be formulated as

$$
(p q)_{c}= \begin{cases}1+\frac{2}{n+\kappa} \max \{p+1, q+1\}, & 0 \leqslant \kappa<+\infty \\ 1, & \kappa=+\infty\end{cases}
$$

That is to say, if $1<\mathrm{pq}<(\mathrm{pq})_{\mathrm{c}}$, any nonnegative nontrivial solution blows up in a finite time, whereas if $\mathrm{pq}>(\mathrm{pq})_{\mathrm{c}}$, there exist both nontrivial nonnegative global solution (with small initial data) and nonnegative blow-up solution (with large initial data). The difference between (1.8) and (1.7) shows that the gradient term can affect the large time behavior of solutions essentially. The technique used in this paper is mainly inspired by $[16,18,21,23]$. To prove the blow-up of solutions, we determine the interactions among the diffusion and the gradient by a series of precise energy integral estimates instead of pointwise comparison principle. For the global existence of nontrivial solutions, we construct a nontrivial global supersolution. Note that neither (1.1) nor (1.2) possesses a self-similar construct, we have to seek a complicated supersolution and do some precise calculations.

The paper is organized as follows. Some preliminaries are listed in Section 2, such as the definition of solution, the well-posedness of the problem (1.1)-(1.3). Several useful auxiliary lemmas are illustrated in Section 3. The blow-up theorems of Fujita type for the problem (1.1)-(1.3) are obtained in Section 4.

\section{Preliminaries}

The subsolutions, supersolutions, as well as solutions to the problem (1.1)-(1.3) are defined as follows.

Definition 2.1. Let $0<\mathrm{T} \leqslant+\infty$. A pair of nonnegative functions $(u, v)$ is called a super (sub) solution to the problem (1.1)-(1.3) in $(0, \mathrm{~T})$, if

$$
u, v \in C\left([0, T), \mathrm{L}_{\text {loc }}^{1}\left(\mathbb{R}^{\mathrm{n}}\right)\right) \cap \mathrm{L}_{\text {loc }}^{\infty}\left(0, \mathrm{~T} ; \mathrm{L}^{\infty}\left(\mathbb{R}^{\mathrm{n}}\right)\right),
$$

and the integral identities

$$
\begin{array}{r}
\int_{0}^{T} \int_{\mathbb{R}^{n}} u(x, t)\left(\frac{\partial \varphi}{\partial t}(x, t)+\Delta \varphi(x, t)-\operatorname{div}(b(|x|) \varphi(x, t) x)\right) d x d t \\
\quad+\int_{0}^{T} \int_{\mathbb{R}^{n}} v^{p}(x, t) \varphi(x, t) d x d t+\int_{\mathbb{R}^{n}} u_{0}(x) \varphi(x, 0) d x \leqslant(\geqslant) 0,
\end{array}
$$

and

$$
\begin{array}{r}
\int_{0}^{T} \int_{\mathbb{R}^{n}} v(x, t)\left(\frac{\partial \psi}{\partial t}(x, t)+\Delta \psi(x, t)-\operatorname{div}(b(|x|) \psi(x, t) x)\right) d x d t \\
\quad+\int_{0}^{T} \int_{\mathbb{R}^{n}} u^{q}(x, t) \psi(x, t) d x d t+\int_{\mathbb{R}^{n}} v_{0}(x) \psi(x, 0) d x \leqslant(\geqslant),
\end{array}
$$

are fulfilled for any $0 \leqslant \varphi, \psi \in C^{2,1}\left(\mathbb{R}^{n} \times[0, T)\right)$ vanishing when $t$ near $T$ or $|x|$ being sufficiently large. $(u, v)$ is called a solution to the problem (1.1)-(1.3) in $(0, T)$, if it is both a supersolution and a subsolution. 
Definition 2.2. A solution $(u, v)$ to the problem (1.1)-(1.3) is said to blow up in a finite time $0<T_{*}<+\infty$, which is called blow-up time, if

$$
\|u(\cdot, t)\|_{L^{\infty}\left(\mathbb{R}^{n}\right)}+\|v(\cdot, t)\|_{L^{\infty}\left(\mathbb{R}^{n}\right)} \rightarrow+\infty, \quad \text { as } \quad t \rightarrow T_{*}^{-} .
$$

Otherwise, $(u, v)$ is said to be global.

We give the existence theorem and the comparison principle to the problem (1.1)-(1.3) as follows without proofs, one can see [13] and the references therein.

Theorem 2.3 (Local existence theorem). If $0 \leqslant u_{0}, v_{0} \in \mathrm{L}_{\text {loc }}^{1}\left(\mathbb{R}^{\mathfrak{n}}\right) \cap \mathrm{L}^{\infty}\left(\mathbb{R}^{\mathfrak{n}}\right)$, then the Cauchy problem (1.1)(1.3) admits at least one solution locally in time.

Theorem 2.4 (Comparison principle). Let $\left(u_{1}, v_{1}\right)$ and $\left(u_{2}, v_{2}\right)$ be two solutions to the system (1.1) and (1.2) in $(0, \mathrm{~T})$ with nonnegative initial data $\mathrm{u}_{1,0}(\mathrm{x}), v_{1,0}(\mathrm{x})$ and $\mathrm{u}_{2,0}(\mathrm{x}), v_{2,0}(\mathrm{x})$, respectively. If $\left(\mathrm{u}_{1,0}, v_{1,0}\right) \leqslant\left(\mathrm{u}_{2,0}, v_{2,0}\right)$ a.e. in $\mathbb{R}^{\mathrm{n}}$, then $\left(\mathrm{u}_{1}, v_{1}\right) \leqslant\left(\mathrm{u}_{2}, v_{2}\right)$ a.e. in $\mathbb{R}^{\mathrm{n}} \times(0, \mathrm{~T})$.

\section{Auxiliary lemmas}

In this section, we illustrate some auxiliary lemmas which will be used to investigate the blow-up property of solutions to the problem (1.1)-(1.3). In this paper, we always assume that $p \geqslant q$ without loss of generality if there is no instruction.

Lemma 3.1. Assume that $0 \leqslant \mathrm{~b} \in \mathrm{C}^{1}([0,+\infty))$ satisfies (1.4) with $0 \leqslant \mathrm{k}<+\infty$. Let $(\mathrm{u}, \mathrm{v})$ be a solution to the problem (1.1)-(1.3). Then there exist three numbers $R_{0}>0, \delta>1$ and $M_{0}>0$ depending only on $n$ and $b$, such that for any $\mathrm{R}>\mathrm{R}_{0}$,

$$
\frac{d}{d t} \int_{\mathbb{R}^{n}} u(x, t) \phi_{R}(|x|) d x \geqslant-M_{0} R^{-2} \int_{B_{\delta R} \backslash B_{R}} u(x, t) \phi_{R}(|x|) d x+\int_{\mathbb{R}^{n}} v^{p}(x, t) \phi_{R}(|x|) d x, \quad t>0,
$$

and

$$
\frac{d}{d t} \int_{\mathbb{R}^{n}} v(x, t) \phi_{R}(|x|) d x \geqslant-M_{0} R^{-2} \int_{B_{\delta R} \backslash B_{R}} v(x, t) \phi_{R}(|x|) d x+\int_{\mathbb{R}^{n}} u^{q}(x, t) \phi_{R}(|x|) d x, \quad t>0,
$$

in the distribution sense, where

$$
\phi_{R}(r)= \begin{cases}h(r), & 0 \leqslant r \leqslant R \\ \frac{1}{2} h(r)\left(1+\cos \frac{(r-R) \pi}{(\delta-1) R}\right), & R<r<\delta R \\ 0, & r \geqslant \delta R\end{cases}
$$

with

$$
h(r)=\exp \left\{\int_{0}^{r} s b(s) d s\right\}, \quad r \geqslant 0,
$$

while $\mathrm{B}_{\mathrm{r}}$ denotes the open ball in $\mathbb{R}^{\mathrm{n}}$ with radius $\mathrm{r}$ and centered at the origin.

Proof. Set $\delta>1$. It is obvious that $\phi_{R} \in C^{1}([0,+\infty)) \cap C^{2}((0, R) \cup(R, \delta R) \cup(\delta R,+\infty))$ with $\phi_{R}^{\prime}(0)=0$. Multiplying (1.1) by $\phi_{R}(|x|)$ and then integrating by parts, one has

$$
\begin{aligned}
\frac{d}{d t} \int_{\mathbb{R}^{n}} u(x, t) \phi_{R}(|x|) d x= & \int_{B_{\delta R}} u(x, t)\left(\Delta \phi_{R}(|x|)-\operatorname{div}\left(b(|x|) \phi_{R}(|x|) x\right)\right) d x \\
& +\int_{\mathbb{R}^{n}} v^{p}(x, t) \phi_{R}(|x|) d x, \quad t>0,
\end{aligned}
$$

in the distribution sense. 
Let us compute $\Delta \phi_{R}(|x|)-\operatorname{div}\left(b(|x|) \phi_{R}(|x|) x\right)$ in $B_{R} \backslash\{0\}$ and $B_{\delta R} \backslash \bar{B}_{R}$, respectively. It is clear that for $0<|x|<R$,

$$
\begin{aligned}
\Delta \phi_{R}(|x|) & =\phi_{R}^{\prime \prime}(|x|)+\frac{n-1}{|x|} \phi_{R}^{\prime}(|x|) \\
& =\left(|x| b^{\prime}(|x|)+b(|x|)+|x|^{2} b^{2}(|x|)+(n-1) b(|x|)\right) \phi_{R}(|x|), \\
\operatorname{div}\left(b(|x|) \phi_{R}(|x|) x\right) & =|x| b^{\prime}(|x|) \phi_{R}(|x|)+|x| b(|x|) \phi_{R}^{\prime}(|x|)+n b(|x|) \phi_{R}(|x|) \\
& =\left(|x| b^{\prime}(|x|)+|x|^{2} b^{2}(|x|)+n b(|x|)\right) \phi_{R}(|x|)
\end{aligned}
$$

thus

$$
\Delta \phi_{\mathrm{R}}(|x|)-\operatorname{div}\left(\mathrm{b}(|x|) \phi_{\mathrm{R}}(|x|) x\right)=0, \quad 0<|x|<\mathrm{R} .
$$

When $R<|x|<\delta R$, a direct calculation gives

$$
\begin{aligned}
\Delta \phi_{R}(|x|) & -\operatorname{div}\left(b(|x|) \phi_{R}(|x|) x\right) \\
= & \phi_{R}^{\prime \prime}(|x|)+\frac{n-1}{|x|} \phi_{R}^{\prime}(|x|)-|x| b^{\prime}(|x|) \phi_{R}(|x|)-|x| b(|x|) \phi_{R}^{\prime}(|x|)-n b(|x|) \phi_{R}(|x|) \\
= & -\frac{\pi}{2(\delta-1) R|x|}\left(|x|^{2} b(|x|)+n-1\right) h(|x|) \sin \frac{(r-R) \pi}{(\delta-1) R}-\frac{\pi^{2}}{2(\delta-1)^{2} R^{2}} h(|x|) \cos \frac{(r-R) \pi}{(\delta-1) R} .
\end{aligned}
$$

It follows from (1.4) that there exists $R_{0}>0$ such that

$$
0<s^{2} b(s)+n-1<k+n, \quad s>R_{0} .
$$

Choose $\delta=\frac{\pi}{n+k}+1$. Then for any $R>R_{0}$, one gets from (3.5) and (3.6) that

$$
\begin{aligned}
\Delta \phi_{R}(|x|)-\operatorname{div}\left(b(|x|) \phi_{R}(|x|) x\right) & \geqslant-\frac{\pi^{2}}{2(\delta-1)^{2} R^{2}} h(|x|)-\frac{\pi^{2}}{2(\delta-1)^{2} R^{2}} h(|x|) \cos \frac{(r-R) \pi}{(\delta-1) R} \\
& =-M_{0} R^{-2} \phi_{R}(|x|), \quad R<|x|<\delta R,
\end{aligned}
$$

with $M_{0}=(\delta-1)^{-2} \pi^{2}$. Substituting (3.4) and (3.7) into (3.3) leads to (3.1). Similarly, one can prove that (3.2) holds.

Remark 3.2. Lemma 3.1 still holds if (1.4) is relaxed by

$$
\varlimsup_{s \rightarrow+\infty} s^{2} b(s)=\kappa \text {. }
$$

Remark 3.3. The proof of Lemma 3.1 is invalid if $\kappa=+\infty$. In this case, both (3.1) and (3.2) hold for any fixed $R>0$, but $\delta>1$ and $M_{0}>0$ depend also on $R$.

To show the existence of a nontrivial global solution to the problem (1.1)-(1.3), we study self-similar supersolutions to the system (1.1) and (1.2) of the form

$$
u(x, t)=(t+\tau)^{-\alpha} U\left((t+\tau)^{-1 / 2}|x|\right), \quad v(x, t)=(t+\tau)^{-\beta} V\left((t+\tau)^{-1 / 2}|x|\right), \quad x \in \mathbb{R}^{n}, t \geqslant 0,
$$

with

$$
\alpha=\frac{p+1}{p q-1}, \quad \beta=\frac{q+1}{p q-1},
$$

and $\tau>0$ will be determined later. If $\mathrm{U}, \mathrm{V} \in \mathrm{C}^{1}([0,+\infty))$ solve

$$
\begin{aligned}
& U^{\prime \prime}(r)+\frac{n-1}{r} U^{\prime}(r)+(t+\tau) r b\left((t+\tau)^{1 / 2} r\right) U^{\prime}(r)+\frac{1}{2} r U^{\prime}(r)+\alpha U(r)+V^{p}(r) \leqslant 0, \quad r>0, \\
& V^{\prime \prime}(r)+\frac{n-1}{r} V^{\prime}(r)+(t+\tau) r b\left((t+\tau)^{1 / 2} r\right) V^{\prime}(r)+\frac{1}{2} r V^{\prime}(r)+\beta V(r)+U^{q}(r) \leqslant 0, \quad r>0,
\end{aligned}
$$

for any $r>0$, respectively. Then $(u, v)$ given by (3.8) is a supersolution to the system (1.1) and (1.2). 
Lemma 3.4. Assume that $\mathrm{b} \in \mathrm{C}^{1}([0,+\infty))$ satisfies (1.4) with $0 \leqslant \mathrm{k} \leqslant+\infty$. Let $\mathrm{pq}>(\mathrm{pq})_{\mathrm{c}}$ and

$$
\mathrm{U}(\mathrm{r})=\mathrm{V}(\mathrm{r})=\varepsilon \mathrm{e}^{-\mathrm{A}(\mathrm{r})}, \quad \mathrm{r} \geqslant 0,
$$

where $\varepsilon>0$ will be determined, while $\mathrm{A} \in \mathrm{C}^{1}([0,+\infty))$ satisfies $\mathrm{A}(0)=0$ and

$$
A^{\prime}(r)= \begin{cases}A_{1} r, & 0 \leqslant r \leqslant l^{2}, \\ A_{2} r+\left(A_{1}-A_{2}\right) \frac{l^{2\left(n+\kappa_{2}\right)}}{r^{n+\kappa_{2}-1}}, & l^{2}<r<l, \\ A_{2} r+\left(A_{1}-A_{2}\right) l^{n+\kappa_{2}} r, & r \geqslant l,\end{cases}
$$

where $0<\mathrm{l}<1$ to be determined,

$$
A_{i}=\frac{2(p+1)}{\left(n+\kappa_{i}\right)\left(p q+(p q)_{c}-2\right)}, \quad i=1,2,
$$

with $-\mathrm{n}<\mathrm{\kappa}_{1}<\mathrm{\kappa}_{2}<\mathrm{\kappa}$ satisfying

$$
\inf \left\{s^{2} b(s): s>0\right\}>\kappa_{1}, \quad\left(n+\kappa_{2}\right)\left(p q+(p q)_{c}-2\right)>4(p+1) .
$$

Then there exist $0<l<1, \tau>0$ and $\varepsilon>0$ such that $(u, v)$ given by (3.8) and (3.11) is a supersolution to the system (1.1) and (1.2).

Proof. For $0<r<l^{2}$ and $t>0$,

$$
\begin{aligned}
U^{\prime \prime}(r) & +\frac{n-1}{r} U^{\prime}(r)+(t+\tau) r b\left((t+\tau)^{1 / 2} r\right) U^{\prime}(r)+\frac{1}{2} r U^{\prime}(r)+\alpha U(r) \\
\leqslant & U(r)\left(-\left(n+\kappa_{0}\right) A_{1}+\frac{p+1}{p q-1}+\left(A_{1}-\frac{1}{2}\right) A_{1} r^{2}\right) \\
& =U(r)\left(-\frac{2\left(\kappa_{0}-\kappa_{1}\right)(p+1)}{\left(n+\kappa_{1}\right)\left(p q+(p q)_{c}-2\right)}+\left(A_{1}-\frac{1}{2}\right) A_{1} r^{2}\right)-U(r) \frac{\left(p q-(p q)_{c}\right)(p+1)}{\left(p q+(p q)_{c}-2\right)(p q-1)}
\end{aligned}
$$

where $\kappa_{0}=\inf \left\{s^{2} b(s): s>0\right\}$. Therefore, there exists $0<l_{1}<1$ such that for any $0<l<l_{1}$,

$$
\begin{aligned}
U^{\prime \prime}(r) & +\frac{n-1}{r} U^{\prime}(r)+(t+\tau) r b\left((t+\tau)^{1 / 2} r\right) U^{\prime}(r)+\frac{1}{2} r U^{\prime}(r)+\alpha U(r) \\
\leqslant & -\frac{\left(p q-(p q)_{c}\right)(p+1)}{2\left(p q+(p q)_{c}-2\right)(p q-1)} U(r), \quad 0<r<l^{2}, t>0
\end{aligned}
$$

From the definition of $A$, one gets

$$
\begin{aligned}
U^{\prime \prime}(r) & +\frac{n+\kappa_{2}-1}{r} U^{\prime}(r)+\frac{1}{2} r U^{\prime}(r)+\alpha U(r) \\
& =U(r)\left(\left(A^{\prime}(r)\right)^{2}-A^{\prime \prime}(r)-\frac{n+\kappa_{2}-1}{r} A^{\prime}(r)-\frac{1}{2} r A^{\prime}(r)+\frac{p+1}{p q-1}\right) \\
& =U(r)\left(\left(A_{2}+\left(A_{1}-A_{2}\right) \frac{l^{2\left(n+\kappa_{2}\right)}}{r^{n+\kappa_{2}}}\right)\left(A_{2}+\left(A_{1}-A_{2}\right) \frac{l^{2\left(n+\kappa_{2}\right)}}{r^{n+\kappa_{2}}}-\frac{1}{2}\right) r^{2}\right)-U(r)\left(n+\kappa_{2}\right) A_{2}+\frac{p+1}{p q-1} \\
& \leqslant U(r)\left(\left(A_{1}-\frac{1}{2}\right) A_{1} r^{2}-\frac{\left(p q-(p q)_{c}\right)(p+1)}{\left(p q+(p q)_{c}-2\right)(p q-1)}\right), \quad l^{2}<r<l,
\end{aligned}
$$

which implies that there exists $0<l_{2}<l_{1}$ such that for any $0<l<l_{2}$,

$$
\mathrm{U}^{\prime \prime}(\mathrm{r})+\frac{\mathrm{n}+\kappa_{2}-1}{\mathrm{r}} \mathrm{U}^{\prime}(\mathrm{r})+\frac{1}{2} r \mathrm{U}^{\prime}(\mathrm{r})+\alpha \mathrm{U}(\mathrm{r}) \leqslant-\frac{\left(p q-(p q)_{c}\right)(p+1)}{2\left(p q+(p q)_{c}-2\right)(p q-1)} \mathrm{U}(\mathrm{r}), \quad \mathrm{l}^{2}<\mathrm{r}<\mathrm{l} .
$$


For $r>l$, it holds that

$$
\begin{aligned}
U^{\prime \prime}(r)+ & \frac{n+\kappa_{2}-1}{r} U^{\prime}(r)+\frac{1}{2} r U^{\prime}(r)+\alpha U(r) \\
= & U(r)\left(\left(A^{\prime}(r)\right)^{2}-A^{\prime \prime}(r)-\frac{n+\kappa_{2}-1}{r} A^{\prime}(r)-\frac{1}{2} r A^{\prime}(r)+\frac{p+1}{p q-1}\right) \\
= & U(r)\left(\left(A_{2}+\left(A_{1}-A_{2}\right) l^{n+\kappa_{2}}\right)\left(A_{2}+\left(A_{1}-A_{2}\right) l^{n+\kappa_{2}}-\frac{1}{2}\right) r^{2}\right. \\
& \left.-\left(n+\kappa_{2}\right)\left(A_{1}-A_{2}\right) l^{n+\kappa_{2}}-\frac{\left(p q-(p q)_{c}\right)(p+1)}{\left(p q+(p q)_{c}-2\right)(p q-1)}\right) \\
\leqslant & U(r)\left(A_{2}+\left(A_{1}-A_{2}\right) l^{n+\kappa_{2}}\right)\left(A_{2}+\left(A_{1}-A_{2}\right) l^{n+\kappa_{2}}-\frac{1}{2}\right) r^{2}-U(r) \frac{\left(p q-(p q)_{c}\right)(p+1)}{\left(p q+(p q)_{c}-2\right)(p q-1)}
\end{aligned}
$$

The choice of $\kappa_{1}, \kappa_{2}$ leads to

$$
\lim _{l \rightarrow 0^{+}}\left(A_{2}+\left(A_{1}-A_{2}\right) l^{n+\kappa_{2}}\right)=A_{2}<\frac{1}{2}
$$

which yields that there exists $0<l_{3}<l_{2}$ such that for any $0<l<l_{3}$,

$$
A_{2}+\left(A_{1}-A_{2}\right) l^{n+k_{2}}<\frac{1}{2}
$$

and thus

$$
U^{\prime \prime}(r)+\frac{n+\kappa_{2}-1}{r} U^{\prime}(r)+\frac{1}{2} r U^{\prime}(r)+\alpha U(r) \leqslant-\frac{\left(p q-(p q)_{c}\right)(p+1)}{2\left(p q+(p q)_{c}-2\right)(p q-1)} U(r), \quad r>l .
$$

Fixing $0<l<l_{3}$, (1.4) ensures that one can choose $\tau>0$ sufficiently large such that

$$
(t+\tau) r b\left((t+\tau)^{1 / 2} r\right) \geqslant \frac{\kappa_{2}}{r}, \quad r>l^{2}, t>0 .
$$

It follows from (3.12), (3.13), (3.14), (3.15) that for $t>0$,

$$
\begin{aligned}
U^{\prime \prime}(r) & +\frac{n-1}{r} U^{\prime}(r)+(t+\tau) r b\left((t+\tau)^{1 / 2} r\right) U^{\prime}(r)+\frac{1}{2} r U^{\prime}(r)+\alpha U(r) \\
& \leqslant U^{\prime \prime}(r)+\frac{n+k_{2}-1}{r} U^{\prime}(r)+\frac{1}{2} r U^{\prime}(r)+\alpha U(r) \\
& \leqslant-\frac{\left(p q-(p q)_{c}\right)(p+1)}{2\left(p q+(p q)_{c}-2\right)(p q-1)} U(r), \quad r \in\left(0, l^{2}\right) \cup\left(l^{2}, l\right) \cup(l,+\infty) .
\end{aligned}
$$

The same as what we calculate above, we can obtain that for $t>0$,

$$
\begin{aligned}
V^{\prime \prime}(r) & +\frac{n-1}{r} V^{\prime}(r)+(t+\tau) r b\left((t+\tau)^{1 / 2} r\right) V^{\prime}(r)+\frac{1}{2} r V^{\prime}(r)+\beta V(r) \\
& \leqslant-\left(\frac{\left(p q-(p q)_{c}\right)(p+1)}{2\left(p q+(p q)_{c}-2\right)(p q-1)}+\frac{p-q}{p q-1}\right) V(r) \\
& \leqslant-\frac{\left(p q-(p q)_{c}\right)(p+1)}{2\left(p q+(p q)_{c}-2\right)(p q-1)} V(r), \quad r \in\left(0, l^{2}\right) \cup\left(l^{2}, l\right) \cup(l,+\infty) .
\end{aligned}
$$

Due to $p, q>1$ and the definition of $A$,

$$
0<K_{0}=\sup _{r>0} e^{-(p-1) A(r)}<+\infty .
$$

Choose $\varepsilon>0$ sufficiently small such that

$$
\max \left\{\varepsilon^{p-1}, \varepsilon^{q-1}\right\} \leqslant \frac{\left(p q-(p q)_{c}\right)(p+1)}{2 K_{0}\left(p q+(p q)_{c}-2\right)(p q-1)} .
$$

Then (3.16) and (3.17) yield that (3.9) and (3.10) for $r \in\left(0, l^{2}\right) \cup\left(l^{2}, l\right) \cup(l,+\infty), t>0$. Since $u, V \in$ $\mathrm{C}^{1,1}([0,+\infty)),(u, v)$ given by (3.8) and (3.11) is a supersolution to the system (1.1) and (1.2). 
Remark 3.5. Lemma 3.4 still holds if $\mathrm{b} \in \mathrm{C}^{1}([0,+\infty))$ and (1.4) are relaxed by that $\mathrm{b}$ is Lipschitz continuous in $[0,+\infty)$ and

$$
\varlimsup_{s \rightarrow+\infty} s^{2} b(s)=\kappa,
$$

respectively.

Also, we need the following elementary inequality.

Lemma 3.6. Assume that $\mathrm{p} \geqslant \mathrm{q}>1$ and $\mathrm{a}, \mathrm{b} \geqslant 0$, then

$$
a^{p}+b^{q} \geqslant \begin{cases}2^{-p}(a+b)^{p}, & \text { if } 0 \leqslant a, b \leqslant 1, \\ 2^{-p}(a+b)^{q}, & \text { otherwise. }\end{cases}
$$

Proof. For $0 \leqslant a, b \leqslant 1$, the convexity of the function $x \mapsto x^{p}$ with $p>1$ yields

$$
(a+b)^{p} \leqslant 2^{p-1}\left(a^{p}+b^{p}\right) \leqslant 2^{p}\left(a^{p}+b^{q}\right),
$$

that is,

$$
a^{p}+b^{q} \geqslant 2^{-p}(a+b)^{p}, \quad 0 \leqslant a, b \leqslant 1 .
$$

Now, we divide the remaining range of $(a, b)$ into the following two parts

$$
D_{1}=\{(a, b) ; a>1, b \geqslant 0\}, \quad D_{2}=\{(a, b) ; 0 \leqslant a \leqslant 1, b>1\} .
$$

If $(a, b) \in D_{1}$, then the convexity of the function $x \mapsto x^{q}$ with $q>1$ ensures

$$
(a+b)^{q} \leqslant 2^{q-1}\left(a^{q}+b^{q}\right) \leqslant 2^{p}\left(a^{p}+b^{q}\right),
$$

i.e.,

$$
a^{p}+b^{q} \geqslant 2^{-p}(a+b)^{q}, \quad(a, b) \in D_{1} .
$$

Finally, if $(a, b) \in D_{2}$, then

$$
\frac{a^{p}+b^{q}}{(a+b)^{q}} \geqslant\left(\frac{b}{a+b}\right)^{q} \geqslant\left(\frac{b}{1+b}\right)^{q} \geqslant\left(\frac{b}{1+b}\right)^{p} .
$$

It follows from $b>1$ that $\frac{b}{1+b} \geqslant \frac{1}{2}$, which together with (3.18), shows

$$
\frac{a^{p}+b^{q}}{(a+b)^{q}} \geqslant 2^{-p}
$$

i.e.,

$$
a^{p}+b^{q} \geqslant 2^{-p}(a+b)^{q}, \quad(a, b) \in D_{2} .
$$

The lemma is proven.

\section{Blow-up theorems of Fujita type}

In this section, we establish the blow-up theorems of Fujita type for the problem (1.1)-(1.3) by using auxiliary lemmas in Section 3. In the future proofs, let $\phi_{R}, h, R_{0}, \delta$ and $M_{0}$ are given in Lemma 3.1.

Firstly, we consider the case $p q<(p q)_{c}$ with $0 \leqslant k<+\infty$.

Theorem 4.1. Assume that $0 \leqslant \mathrm{~b} \in \mathrm{C}^{1}([0,+\infty))$ satisfies (1.4) with $0 \leqslant \mathrm{k}<+\infty$. Let $\mathrm{pq}<(\mathrm{pq})_{\mathrm{c}}$. Then for any nontrivial $0 \leqslant u_{0}, v_{0} \in \mathrm{L}_{\text {loc }}^{1}\left(\mathbb{R}^{\mathfrak{n}}\right) \cap \mathrm{L}^{\infty}\left(\mathbb{R}^{\mathrm{n}}\right)$, the solution to the problem (1.1)-(1.3) must blow up in a finite time. 
Proof. Owing to $0 \leqslant k<+\infty$ and $p q<(p q)_{c}$,

$$
\kappa<\frac{2(p+1)}{p q-1}-n
$$

Fix $\tilde{K}$ to satisfy

$$
\kappa<\tilde{\kappa}<\frac{2(p+1)}{p q-1}-n .
$$

By (1.4) and (4.1), there exists $R_{1}>0$ such that

$$
0 \leqslant s^{2} b(s)<\tilde{k}, \quad s>R_{1} .
$$

For any $R>R_{1}$, one gets

$$
\begin{aligned}
0 \leqslant \phi_{R}(|x|) & \leqslant h(|x|) x_{[0, \delta R]}(|x|) \leqslant \exp \left\{\int_{0}^{\delta R} s b(s) d s\right\} x_{[0, \delta R]}(|x|) \\
& \leqslant \exp \left\{\int_{0}^{R_{1}} s b(s) d s\right\} \exp \left\{\tilde{\kappa} \int_{R_{1}}^{\delta R} \frac{1}{s} d s\right\} x_{[0, \delta R]}(|x|)=M R^{\tilde{\kappa}} \chi_{[0, \delta R]}(|x|), \quad|x| \geqslant 0,
\end{aligned}
$$

where $\chi_{[0, \delta R]}$ is the characteristic function of the interval $[0, \delta R]$, while $M>0$ depends only on $n, b, R_{1}, \delta$ and $\tilde{k}$. Let $(u, v)$ be the solution to the problem (1.1)-(1.3), and denote

$$
w_{R}(t)=\int_{\mathbb{R}^{n}}\left(u(x, t)+R^{\theta} v(x, t)\right) \phi_{R}(|x|) d x, \quad t \geqslant 0,
$$

with

$$
\theta=-\frac{(p-q)(n+\tilde{\kappa})}{p+1} .
$$

For any $R>\max \left\{R_{0}, R_{1}\right\}$, Lemma 3.1 shows

$$
\frac{d}{d t} w_{R}(t) \geqslant-M_{0} R^{-2} w_{R}(t)+R^{\theta} \int_{\mathbb{R}^{n}} u^{q}(x, t) \phi_{R}(|x|) d x+\int_{\mathbb{R}^{n}} v^{p}(x, t) \phi_{R}(|x|) d x, \quad t>0 .
$$

The Hölder inequality and (4.2) yield

$$
\begin{aligned}
\int_{\mathbb{R}^{n}} u(x, t) \phi_{R}(|x|) d x & \leqslant\left(\int_{\mathbb{R}^{n}} \phi_{R}(|x|) d x\right)^{(q-1) / q}\left(\int_{\mathbb{R}^{n}} u^{q}(x, t) \phi_{R}(|x|) d x\right)^{1 / q} \\
& \leqslant\left(M R^{\tilde{k}} \int_{B_{\delta R}} d x\right)^{(q-1) / q}\left(\int_{\mathbb{R}^{n}} u^{q}(x, t) \phi_{R}(|x|) d x\right)^{1 / q} \\
& =M_{1}^{(q-1) / q} R^{(n+\tilde{\kappa})(q-1) / q}\left(\int_{\mathbb{R}^{n}} u^{q}(x, t) \phi_{R}(|x|) d x\right)^{1 / q}, \quad t>0,
\end{aligned}
$$

with $M_{1}=M \omega_{n} \delta^{n}>0$ and $\omega_{n}$ is the volume of the unit ball in $\mathbb{R}^{n}$, which implies

$$
\int_{\mathbb{R}^{n}} u^{q}(x, t) \phi_{R}(|x|) d x \geqslant M_{1}^{1-q} R^{(n+\tilde{k})(1-q)}\left(\int_{\mathbb{R}^{n}} u(x, t) \phi_{R}(|x|) d x\right)^{q}, \quad t>0 .
$$

Similarly, one can prove that

$$
\int_{\mathbb{R}^{n}} v^{p}(x, t) \phi_{R}(|x|) d x \geqslant M_{1}^{1-p} R^{(n+\tilde{\kappa})(1-p)}\left(\int_{\mathbb{R}^{n}} v(x, t) \phi_{R}(|x|) d x\right)^{p}, \quad t>0 .
$$


Substituting (4.4) and (4.5) into (4.3) shows

$$
\begin{aligned}
\frac{d}{d t} w_{R}(t) \geqslant & -M_{0} R^{-2} w_{R}(t)+M_{1}^{1-q} R^{(n+\tilde{\kappa})(1-q)+\theta}\left(\int_{\mathbb{R}^{n}} u(x, t) \phi_{R}(|x|) d x\right)^{q} \\
& +M_{1}^{1-p} R^{(n+\tilde{\kappa})(1-p)-p \theta}\left(\int_{\mathbb{R}^{n}} R^{\theta} v(x, t) \phi_{R}(|x|) d x\right)^{p}, \quad t>0 .
\end{aligned}
$$

It follows from

$$
(n+\tilde{\kappa})(1-q)+\theta=(n+\tilde{\kappa})(1-p)-p \theta=-\frac{(p q-1)(n+\tilde{\kappa})}{p+1},
$$

and Lemma 3.6 that (4.6) leads to

$$
\begin{aligned}
\frac{d}{d t} w_{R}(t) \geqslant & -M_{0} R^{-2} w_{R}(t)+M_{2} R^{-(p q-1)(n+\tilde{\kappa}) /(p+1)} \\
& \cdot\left\{\left(\int_{\mathbb{R}^{n}} u(x, t) \phi_{R}(|x|) d x\right)^{q}+\left(\int_{\mathbb{R}^{n}} R^{\Theta} v(x, t) \phi_{R}(|x|) d x\right)^{p}\right\} \\
\geqslant & -M_{0} R^{-2} w_{R}(t)+2^{-p} M_{2} R^{-(p q-1)(n+\tilde{\kappa}) /(p+1)} \cdot \min \left\{w_{R}^{p}(t), w_{R}^{q}(t)\right\} \\
= & w_{R}(t)\left(-M_{0} R^{-2}+2^{-p} M_{2} R^{-(p q-1)(n+\tilde{\kappa}) /(p+1)} \cdot \min \left\{w_{R}^{p-1}(t), w_{R}^{q-1}(t)\right\}\right), \quad t>0,
\end{aligned}
$$

with $M_{2}=\min \left\{M_{1}^{1-p}, M_{1}^{1-q}\right\}$. Note that (4.1) implies

$$
-\frac{(p q-1)(n+\tilde{k})}{p+1}>-2
$$

while $w_{R}(0)$ is nondecreasing with respect to $R \in(0,+\infty)$ and

$$
\sup \left\{w_{R}(0): R>0\right\}>0 .
$$

Therefore, there exists $R_{2}>0$ such that for any $R>R_{2}$,

$$
M_{0} R^{-2} \leqslant 2^{-(p+1)} M_{2} R^{-(p q-1)(n+\tilde{\kappa}) /(p+1)} \cdot \min \left\{w_{R}^{p-1}(0), w_{R}^{q-1}(0)\right\} .
$$

Fix $R>\max \left\{R_{0}, R_{1}, R_{2}\right\}$. Then (4.7) and (4.8) yield

$$
\frac{d}{d t} w_{R}(t) \geqslant 2^{-(p+1)} M_{2} R^{-(p q-1)(n+\tilde{\kappa}) /(p+1)} \cdot \min \left\{w_{R}^{p}(t), w_{R}^{q}(t)\right\}, \quad t>0 .
$$

Since $p, q>1$, there exists $T_{*}>0$ such that

$$
w_{R}(t)=\int_{\mathbb{R}^{n}}\left(u(x, t)+R^{\theta} v(x, t)\right) \phi_{R}(|x|) d x \rightarrow+\infty \quad \text { as } \quad t \rightarrow T_{*}^{-} .
$$

From supp $\phi_{R}(|x|)=\bar{B}_{\delta R}$, one gets

$$
\|\mathrm{u}(\cdot, \mathrm{t})\|_{\mathrm{L}^{\infty}\left(\mathbb{R}^{\mathrm{n}}\right)}+\|v(\cdot, \mathrm{t})\|_{\mathrm{L}^{\infty}\left(\mathbb{R}^{\mathrm{n}}\right)} \rightarrow+\infty \quad \text { as } \mathrm{t} \rightarrow \mathrm{T}_{*}^{-} .
$$

That is to say, $(u, v)$ blows up in a finite time.

Turn to the case $p q>(p q)_{c}$ with $0 \leqslant k \leqslant+\infty$.

Theorem 4.2. Assume that $0 \leqslant \mathrm{~b} \in \mathrm{C}^{1}([0,+\infty))$ satisfies (1.4) with $0 \leqslant \mathrm{k} \leqslant+\infty$. Let $\mathrm{pq}>(\mathrm{pq})_{\mathrm{c}}$. Then there exist both nontrivial global and blow-up solutions to the problem (1.1)-(1.3). 
Proof. The comparison principle and Lemma 3.4 yield that the problem (1.1)-(1.3) with small initial data admits a nontrivial global solution. Let us show the existence of blow-up solution to the problem (1.1)(1.3) with large initial data.

Fix $R>R_{0}$ with $R_{0}$ given in Lemma 3.1 and let $(u, v)$ be the solution to the problem (1.1)-(1.3). Denote

$$
\tilde{w}_{R}(t)=\int_{\mathbb{R}^{n}}(u(x, t)+v(x, t)) \phi_{R}(|x|) d x, \quad t \geqslant 0 .
$$

It follows from Lemma 3.1 (the case $0 \leqslant k<+\infty$ ), Remark 3.3 (the case $\kappa=+\infty$ ), the Hölder inequality and Lemma 3.6 that

$$
\begin{aligned}
\frac{d}{d t} \tilde{w}_{R}(t) \geqslant & -M_{0} R^{-2} \tilde{w}_{R}(t)+\int_{\mathbb{R}^{n}} u^{q}(x, t) \phi_{R}(|x|) d x+\int_{\mathbb{R}^{n}} v^{p}(x, t) \phi_{R}(|x|) d x \\
\geqslant & -M_{0} R^{-2} \tilde{w}_{R}(t)+\left(\int_{\mathbb{R}^{n}} \phi_{R}(|x|) d x\right)^{1-q}\left(\int_{\mathbb{R}^{n}} u(x, t) \phi_{R}(|x|) d x\right)^{q} \\
& +\left(\int_{\mathbb{R}^{n}} \phi_{R}(|x|) d x\right)^{1-p}\left(\int_{\mathbb{R}^{n}} v(x, t) \phi_{R}(|x|) d x\right)^{p} \\
\geqslant & -M_{0} R^{-2} \tilde{w}_{R}(t)+M_{3}\left(\int_{\mathbb{R}^{n}} u(x, t) \phi_{R}(|x|) d x\right)^{q}+M_{3}\left(\int_{\mathbb{R}^{n}} v(x, t) \phi_{R}(|x|) d x\right)^{p} \\
\geqslant & -M_{0} R^{-2} \tilde{w}_{R}(t)+2^{-p} M_{3} \cdot \min \left\{\tilde{w}_{R}^{p}(t), \tilde{w}_{R}^{q}(t)\right\} \\
= & \tilde{w}_{R}(t)\left(-M_{0} R^{-2}+2^{-p} M_{3} \cdot \min \left\{\tilde{w}_{R}^{p-1}(t), \tilde{w}_{R}^{q-1}(t)\right\}\right), \quad t>0,
\end{aligned}
$$

where $\phi_{R}$ and $\delta$ are mentioned in Lemma 3.1, and

$$
M_{3}=\min \left\{\left(\int_{\mathbb{R}^{n}} \phi_{R}(|x|) d x\right)^{1-q},\left(\int_{\mathbb{R}^{n}} \phi_{R}(|x|) d x\right)^{1-p}\right\},
$$

is a positive constant depended only on $n, \delta, p$, q and $R$. If $\left(u_{0}, v_{0}\right)$ is so large that

$$
2^{-(p+1)} M_{3} \cdot \min \left\{\tilde{w}_{R}^{p-1}(0), \tilde{w}_{R}^{q-1}(0)\right\} \geqslant M_{0} R^{-2},
$$

then (4.9) leads to

$$
\frac{d}{d t} \tilde{w}_{R}(t) \geqslant 2^{-(p+1)} M_{3} \cdot \min \left\{\tilde{w}_{R}^{p}(t), \tilde{w}_{R}^{q}(t)\right\}, \quad t>0 .
$$

By the same argument as in the end of the proof of Theorem 4.1, one can show that $(u, v)$ must blow up in a finite time.

Remark 4.3. Theorems 4.1 and 4.2 still hold if $\mathrm{b} \in \mathrm{C}^{1}([0,+\infty))$ is relaxed by that $\mathrm{b}$ is Lipschitz continuous in $[0,+\infty)$.

Remark 4.4. According to Remarks 3.2 and 3.5, Theorem 4.1 still holds if (1.4) is relaxed by

$$
\varlimsup_{s \rightarrow+\infty} s^{2} b(s)=\kappa,
$$

while Theorem 4.2 still holds if (1.4) is relaxed by

$$
\varliminf_{s \rightarrow+\infty} s^{2} b(s)=\kappa .
$$

\section{Acknowledgment}

This work is supported by the National Natural Science Foundation of China (No. 11571137 and 11601182). 


\section{References}

[1] K. Deng, H. A. Levine, The role of critical exponents in blow-up theorems: the sequel, J. Math. Anal. Appl., 243 (2000), 85-126. 1

[2] M. Escobedo, M. A. Herrero, Boundedness and blow up for a semilinear reaction-diffusion system, J. Differential Equations, 89 (1991), 176-202. 1, 1

[3] H. Fujita, On the blowing up of solutions of the Cauchy problem for $\mathrm{u}_{\mathrm{t}}=\Delta \mathrm{u}+\mathrm{u}^{1+\alpha}$, J. Fac. Sci. Univ. Tokyo Sect. I, 13 (1966), 109-124. 1

[4] W. Guo, M. Lei, Critical Fujita curves for a coupled reaction-convection-diffusion system with singular coefficients, J. Jilin Univ. Sci., 54 (2016), 183-188. 1, 1

[5] W. Guo, X. Wang, M. Zhou, Asymptotic behavior of solutions to a class of semilinear parabolic equations, Bound. Value Probl., 2016 (2016), 9 pages. 1

[6] K. Hayakawa, On nonexistence of global solutions of some semilinear parabolic equations, Proc. Japan Acad., 49 (1973), 503-505. 1

[7] K. Kobayashi, T. Siaro, H. Tanaka, On the blowing up problem for semilinear heat equations, J. Math. Soc. Japan, 29 (1977), 407-424. 1

[8] H. A. Levine, The role of critical exponents in blow-up theorems, SIAM Rev., 32 (1990), 262-288. 1

[9] H.-L. Li, X.-Y. Wang, Y.-Y. Nie, H. He, Asymptotic behavior of solutions to a degenerate quasilinear parabolic equation with a gradient term, Electron. J. Differential Equations, 2015 (2015), 12 pages.

[10] P. Meier, On the critical exponent for reaction-diffusion equations, Arch. Rational Mech. Anal., 109 (1990), 63-71. 1

[11] Y.-W. Qi, The critical exponents of parabolic equations and blow-up in $\mathrm{R}^{n}$, Proc. Roy. Soc. Edinburgh Sect. A, 128 (1998), 123-136.

[12] Y.-W. Qi, M.-X. Wang, Critical exponents of quasilinear parabolic equations, J. Math. Anal. Appl., 267 (2002), $264-280$. 1

[13] P. Quittner, P. Souplet, Superlinear parabolic problems: blow-up, global existence and steady states, Birkhäuser, Basel, (2007). 2

[14] R. Suzuki, Existence and nonexistence of global solutions to quasilinear parabolic equations with convection, Hokkaido Math. J., 27 (1998), 147-196. 1, 1

[15] C.-P. Wang, Asymptotic behavior of solutions to a class of semilinear parabolic equations with boundary degeneracy, Proc. Amer. Math. Soc., 141 (2013), 3125-3140.

[16] C.-P. Wang, S. Zheng, Critical Fujita exponents of degenerate and singular parabolic equations, Proc. Roy. Soc. Edinburgh Sect. A, 136 (2006), 415-430. 1

[17] C.-P. Wang, S. Zheng, Fujita-type theorems for a class of nonlinear diffusion equations, Differential Integral Equations, 26 (2013), 555-570.

[18] C.-P. Wang, S. Zheng, Z. Wang, Critical Fujita exponents for a class of quasilinear equations with homogeneous Neumann boundary data, Nonlinearity, 20 (2007), 1343-1359. 1, 1, 1

[19] F. B. Weissler, Existence and non-existence of global solutions for semilinear equation, Israel J. Math., 38 (1981), 29-40. 1

[20] S. Zheng, X.-F. Song, Z.-X. Jiang, Critical Fujita exponents for degenerate parabolic equations coupled via nonlinear boundary flux, J. Math. Anal. Appl., 298 (2004), 308-324. 1

[21] S. Zheng, C.-P. Wang, Large time behaviour of solutions to a class of quasilinear parabolic equations with convection terms, Nonlinearity, 21 (2008), 2179-2200. 1, 1, 1

[22] M.-J. Zhou, H.-L. Li, W. Guo, X. Zhou, Critical Fujita exponents to a class of non-Newtonian filtration equations with fast diffusion, Bound. Value Probl., 2016 (2016), 16 pages.

[23] Q. Zhou, Y.-Y. Nie, X.-Y. Han, Large time behavior of solutions to semilinear parabolic equations with gradient, J. Dyn. Control Syst., 22 (2016), 191-205. 1, 1, 1 